\title{
Evaluation of Sealing Ability of Zinc Oxide Nano-Particles Versus Conventional Zinc Oxide in Endodontically Treated Teeth (SEM study)
}

\author{
Rana Hany Yaseen ${ }^{(1)}$, Mohsen Nour El-Din ${ }^{(2)}$, Mahmoud Hassan Mohamed ${ }^{(3)}$
}

Codex : $51 / 1810$

azhardentj@azhar.edu.eg

http://adjg.journals.ekb.eg

\section{KEYWORDS}

$\mathrm{ZnO}$ nanosealer, Conventional

ZNO, Micoleakage

\begin{abstract}
Purpose: The aim of this study was directed to compare the sealing ability of two materials which are conventional zinc oxide and zinc oxide nanoparticles by measuring microleakage of both materials by stereo microscope and scanning electron microscope. Materials and Methods: Seventy extracted single-rooted human teeth with complete roots, fully formed apices and present a single canal without calcification were used. The crowns of all teeth were removed using a water-cooled, slow-speed diamond precision disc, so as to adjust the length of the roots to a standardized length of $16 \mathrm{~mm}$. The samples were randomly divided into two main groups each one contains 35 samples according to the sealer used as follow: Group I: Conventional zinc oxide sealer \& Group II: Zinc oxide nano-particles. Each main group was divided according to evaluation method as follow: Thirty roots were randomly selected from each group for the evaluation of sealing ability using dye penetration method to measure microleakage using stereo microscope and the rest (5 roots) were used in the SEM for representative evaluation of sealing ability of each sealer. In the stereo microscope part, the samples were further divided into three subdivisions according to the time of incubation period one week, one month and three months. Results: As for Dye leakage results of the sealing ability, zno nano-powder showed the statistically significantly highest sealing throughout all periods of examination, While conventional zno showed the statistically significantly lowest sealing throughout all periods of examination. For both zno nano sealer and conventional zinc oxide samples, the highest mean value of leakage was found in (After 3 months) followed by (After 1 week) while the least mean value of leakage was found in (After 1 month). Conclusion: Zinc Oxide nano particles based sealer has higher sealing ability than conventional zinc oxide based sealer.Nano sized particles allow more adaptation and penetration into dentinal tubules than macro sized one.
\end{abstract}

- Paper extracted from Master thesis entiteled "Evaluation Of Sealing Ability Of Zinc Oxide Nano-Particles Versus Conventional Zinc Oxide in Endodontically Treated Teeth"

1. Dentist at Ministry of Health, Egypt.

2. Professor of Endodontics, Faculty of Dental Medicine for girls, Al-Azhar University.

3. Professor and Head of Endodontic department, Faculty of Dental Medicine for Boys, Al Azhar University, Assuit branch. 


\section{INTRODUCTION}

The main aim of root canal therapy is the removal of microbial contaminants in conjunction with the total closure of the root canal system. Root canal sealers along with solid core material plays a major role in achieving the three dimensional sealing of the root canal system. These sealers are binding agents which are used to adapt the rigid gutta percha to canal walls and to fill up the voids, accessory canals and irregularities within the canal. Root canal sealers, although used only as adjunctive materials in the obturation of root canals, have been shown to influence the outcome of endodontic treatment. A perfect combination of sealing ability and biocompatibility is what an ideal root canal sealer should possess ${ }^{(1)}$. Incomplete obturation of the root canal accounts for $58 \%$ of endodontic failures. The incomplete obturation may be because of incomplete instrumentation or improper obturation technique ${ }^{(2)}$.

The sealers used should fill the discrepancies between the canal wall and the gutta-percha; act as a lubricant and aid in seating the gutta-percha cones. The sealers should also fill the patent accessory and lateral canals, entomb the bacteria present within the dentinal tubule and allow for the repair of the periapical tissue $^{(3)}$.

This study discusses the current concepts in the usage of different sealers in endodontic therapy and their comparison in order to draw some clinical inferences. Ideally, the root canal sealer should be capable of creating an effective bond between the core material and the dentine of the root canal thus preventing leakage ${ }^{(4)}$.

A great variety of endodontic sealers are available commercially and they are divided into different groups according to their chemical composition. A common cause of failure of root canal treatment is due to the inability to seal the accessory canal in most cases, as the microleakage, whether apical or coronal may cause failure of root canal therapy ${ }^{(5)}$.
Being a combination of zinc oxide powder and eugenol liquid, zinc oxide eugenol (ZOE) cement is one of the most applied materials in the field of dentistry. This cement may be used as the base under direct restorations, temporary fillings, root canal sealer, orthodontic band cement or periodontal and surgical pack. The most remarkable reason for paying attention to this cement is related to its unique biological properties including its sedative impact on exposed dentinal tubules, biocompatibility, neutral $\mathrm{pH}$, anti-microbial and anti- cariogenic properties, anti-inflammatory effect and palliative character of this solution ${ }^{(6)}$.

Currently, paralleled to recent technological advances, it is possible to provide zinc oxide powder through nanotechnology. Nanotechnology is a research hot spot in modern materials science. It is considered as the synthesis, characterization, and exploration of materials in the nanometer region. In this technology, the pertinent materials are those whose structures exhibit new and considerably enhanced physicochemical and biological properties as a result of the nanoscale size ${ }^{(7)}$.

This nanoscale size generally confers larger surface areas to nanoparticles (NPs) compared with macro-sized particles. The intrinsic properties of metal NPs such as zinc oxide $(\mathrm{ZnO})$ and silver are mostly characterized by their size, composition, crystallinity and morphology. Reducing the size to nanoscale can modify their chemical, mechanical, electrical, structural, morphological, and optical properties. These modified features allow the NPs to interact in a unique manner with cell biomolecules and thus facilitate the physical transfer of NPs into the inner cellular structures. Thus, nanomaterials have witnessed recently significant importance in the basic sciences ${ }^{\left({ }^{(8)}\right.}$.

Microleakage tests are the most favourable in vitro methods to evaluate the sealing efficiency of a root canal fillings or sealers. Various methods such as dye penetration test, fluid filtration test, radioactive isotopes, bacterial penetration and electrochemical leakage test are present. The most 
common method is dye penetration due to its sensitivity, ease of use and convenience ${ }^{(9)}$.

\section{MATERIALS AND METHODS}

In this study, 70 extracted single-rooted human teeth with complete roots, fully formed apices and present a single canal without calcification were used. The teeth were macroscopically examined and radiographed in mesiodistal and buccolingual direction, teeth with root caries, external resorption, fracture or cracks were excluded and replaced by another one.

Soft tissues and debris were removed by leaving the teeth in Naocl for 30 minutes and calculus were removed from the tooth surface using fine stone in low speed hand piece with attention not to damage the root surface. The teeth were stored in fresh water and $0.5 \%$ sodium Azide until use.The crowns of all teeth were removed using a water-cooled, slow-speed diamond precision disc, so as to adjust the length of the roots to a standardized length of $16 \mathrm{~mm}$.

Canal patency and working length were established by inserting K-file \#15 with its tip visualized at the apical foramen and subtracting 1-mm from this length.

Protaper Universal rotary NiTi files were used in a crown-down manner for root canal preparation with a 16:1 reduction handpiece that was powered by a torque-controlled electric motor; at a rotational speed of $300 \mathrm{rpm}$ and a torque-control of $2.6 \mathrm{~N} / \mathrm{cm}$. The protaper universal rotary files were used with slow downward movement in a free progression without pressure as follows:

- SX file was inserted in the canal until resistance was encountered to flare the orifice of the root with brushing action on the walls

- Followed by two shaping files (S1 and S2) were used to full working length with brushing action on the walls
- Three finishing files (F1, F2 and F3) for apical finishing used with up and down movement (no brushing action) to full working lengh.

The master apical file was chosen to be a size F3 for all specimens. In conjunction with $2 \mathrm{ml}$ of Normal saline solution (Sodium Chloride $0.9 \%$ ) irrigation between each file and $3 \mathrm{ml}$ of the chelating solution was applied for 1 minute then after complete mechanical preparation the canals were irrigated with $3 \mathrm{ml}$ distilled water for one minute then the root canals were dried with absorbent paper points.

\section{Sample grouping:}

The samples were randomly divided into two main groups each one contains 35 samples according to the sealer used as follow:

Group I: Conventional zinc oxide sealer \& Group II: Zinc oxide nano-particles.

Each main group was divided according to evaluation method as follow:

Thirty roots were randomly selected from each group for the evaluation of sealing ability using dye penetration method to measure microleakage using stereo microscope and the rest (5 roots) were used in the SEM for representative evaluation of sealing ability of each sealer.

In the stereo microscope part, the samples were further divided into three subdivisions according to the time of incubation period as follow:

\section{Subgroup (A) the first examined group 10 samples (storage time one week):}

Both materials were applied by one calibrated operator in strict adherence to the manufacturers' recommendations. Following root filling procedures, canal openings were sealed with $\mathrm{Cavit}^{\mathrm{TM}}-\mathrm{G}$. The specimens were stored at $37^{\circ} \mathrm{C}$ and $100 \%$ humidity in an incubator for 1 week to allow complete setting of the tested materials. 


\section{Sub group (B) the second examined group 10 samples (storage time 1 month):}

On completion of instrumentation, the same procedure as subgroup (A) was done and the specimens were stored at $37^{\circ} \mathrm{C}$ and $100 \%$ humidity in an incubator for one month to allow complete setting of the tested materials.

Sub group (C) the third examined group 10 samples (storage time 3 months):

On completion of instrumentation, the same procedure as subgroups (A) \& (B) was done, The specimens were stored at $37^{\circ} \mathrm{C}$ and $100 \%$ humidity in an incubator for 3 months to allow complete setting of the tested materials.

\section{Obturation of samples:}

After completion of instrumentation, the root canals were then dried with paper points corresbonding to the same size of master cone In this study we use single cone obturation technique (Protaper Gutta Percha point size F3), the master cone was inserted and tried into the canal to the full working length and verified for a tight fit.

Manufacturer instructions were followed to obtain a homogenous creamy mix of both sealeres using metal spatula and dry glass slab.

The sealers were loaded and introduced to the canal using $\mathrm{K}$ file size 30 , the file was twirled counter clockwise, pumped up and down and wiped against the walls to apply the sealer all over canal wall, then the prefitted master cone dipped into mixing sealer and inserted very slowly into the canal to the full working length, then hand plugger was used to remove excess gutta percha from the orifice of the canal.

After the obturation, periapical radiographs of each filled root were taken from both labial and proximal views to assess adequacy of obturation. Following root filling procedures, canal openings were sealed with Cavit ${ }^{\mathrm{TM}}-\mathrm{G}$.

\section{Sample preparation for evaluating the sealing ability of each sealer using stereo microscope:}

The samples were dried with an air jet for two minutes and two layers of nail varnish was applied with two hours intervals between each layer on the external root surfaces except at the apical $2 \mathrm{~mm}$ to allow dye penetration into the accessory and lateral canals. After the storage period the samples were suspended in closed tube containing $1 \%$ methylene blue dye ph 7 for 72 hours. After three days, the nail varnish was scrapped off from the root surface, the roots were mounted on resin blocks and longitudinally sectioned for stereomicroscopic imaging of the vertical dye penetration at $30 \mathrm{X}$ magnification. The samples were then observed under stereomicroscope. The dye leakage was measured with a millimeter scale from the apical constriction to the longest point of dye penetration along the canal walls and obturation material itself. The obtained results were submitted for statistical analysis.

\section{Evaluation of sealing ability using Scanning elec- tron microscope (SEM):}

Five samples from each group were randomly selected. Specimens were prepared for SEM evaluation as follow: samples were grooved longitudinally on the external surfaces (buccal \&lingual) with a diamond disk, avoiding penetration of the root canals. The teeth were then carefully split with a hammer and chisel into two halves, The half which retained the obturation material was chosen for examination under SEM, then mounted on aluminum stubs.

The samples were examined with the scanning electron microscope at different magnifications $300 \mathrm{X}$ and 200X, using an acceleration voltage of $30 \mathrm{KV}$. The specimens were examined from coronal, middle and apical thirds and micrographs were taken.

\section{Statistical Analysis:}

The collected data were tabulated and the mean and standard deviation values were calculated 
for each group in each test. Viable counts of antibacterial activity were transformed to their $\log 10$ values. Data were explored for normality using Kolmogorov-Smirnov and Shapiro-Wilk tests and showed parametric (normal) distribution.

Independent sample t-test was used to compare between two groups in nonrelated samples. Repeated measure ANOVA was used to compare between more than two groups in related samples. Paired sample t-test was used to compare between two groups in related samples.

The significance level was set at $\mathrm{P} \leq 0.05$. Statistical analysis was performed with IBM ${ }^{\circledR}$ SPSS ${ }^{\circledR}$ Statistics Version 20 for Windows.

\section{RESULTS}

Part 1: Evaluation of sealing ability using stereo microscope:

\section{I-Relation between different time periods in each group:}

\section{a) Conventional $\mathrm{ZnO}$ :}

The highest mean value of leakage was found in (After 3 months) followed by (After 1 week) while the least mean value of leakage was found in (After 1 month).

\section{b) ZnO nano particles:}

The highest mean value of leakage was found in (After 3 months) followed by (After 1 week) while the least mean value of leakage was found in (After 1 month)

II) Relation between different groups in each time period:

a) After 1 week: The highest mean value of leakage was found in (Conventional $\mathrm{ZnO}$ ), while the least mean value of leakage was found in (ZnO nano particles).

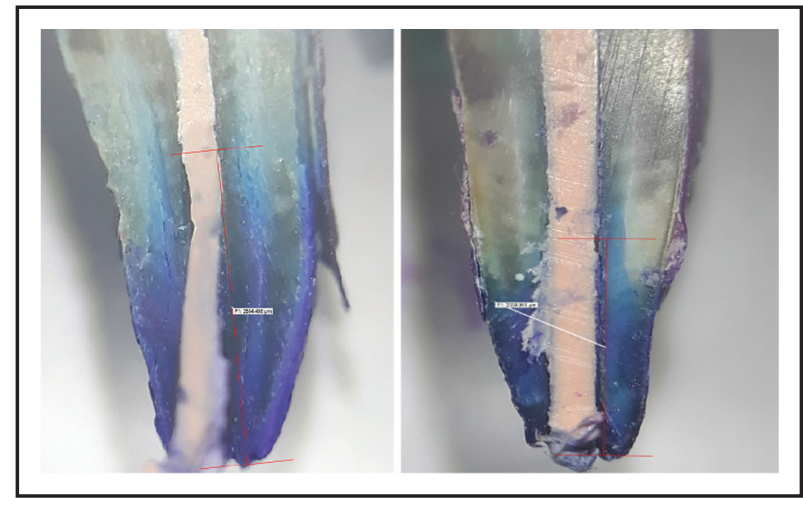

Figure (1): Image under stereo microscope representing dye leakage in conventional zinc oxide and nano zinc oxide based sealers after one week incubation period.

b) After 1 month: The highest mean value of leakage was found in (Conventional $\mathrm{ZnO}$ ), while the least mean value of leakage was found in ( $\mathrm{ZnO}$ nano particles).

\section{c) After 3 months:}

The highest mean value of leakage was found in (Conventional $\mathrm{ZnO}$ ), while the least mean value of leakage was found in ( $\mathrm{ZnO}$ nano particles).

Table (1): The mean, standard deviation (SD) values of Sealing ability of different groups.

\begin{tabular}{|c|c|c|c|c|c|}
\hline \multirow{3}{*}{ Variables } & \multicolumn{5}{|c|}{ Sealing ability } \\
\hline & \multicolumn{2}{|c|}{ Conventional $\mathrm{ZnO}$} & \multicolumn{2}{|c|}{ ZnO nano particles } & \multirow{2}{*}{$p$-value } \\
\hline & Mean & SD & Mean & SD & \\
\hline After 1 week & $3328.08^{\mathrm{aA}}$ & 773.51 & $2718.64^{\mathrm{aA}}$ & 889.46 & $0.093 \mathrm{~ns}$ \\
\hline After 1 months & $2379.58^{\mathrm{bA}}$ & 574.41 & $2008.57^{\mathrm{bA}}$ & 731.82 & $0.236 \mathrm{~ns}$ \\
\hline After 3 months & $3505.58^{\mathrm{aA}}$ & 409.72 & $2892.92^{\text {ав }}$ & 847.03 & $0.033 *$ \\
\hline p-value & \multicolumn{2}{|c|}{$0.002 *$} & \multicolumn{2}{|c|}{$0.005^{*}$} & \\
\hline
\end{tabular}


Part 2 : Evaluation of sealing ability using Scanning Electron Microscope:

Examination of the samples with SEM revealed that, Zinc oxide nano particles sealer was well adapted and well sealed to both dentine and gutta percha, penetrated into the dentinal tubules than conventional zinc oxide which showed some gaps at both the sealer-dentin and sealer-gutta percha interfaces. In both sealers sealing and penetration were more prominent in coronal and middle thirds than in apical third of root canals where there was only a slight gap between root dentin and sealer . Zno nano particles appears to have a granular appearance of varying sizes on the root canal.
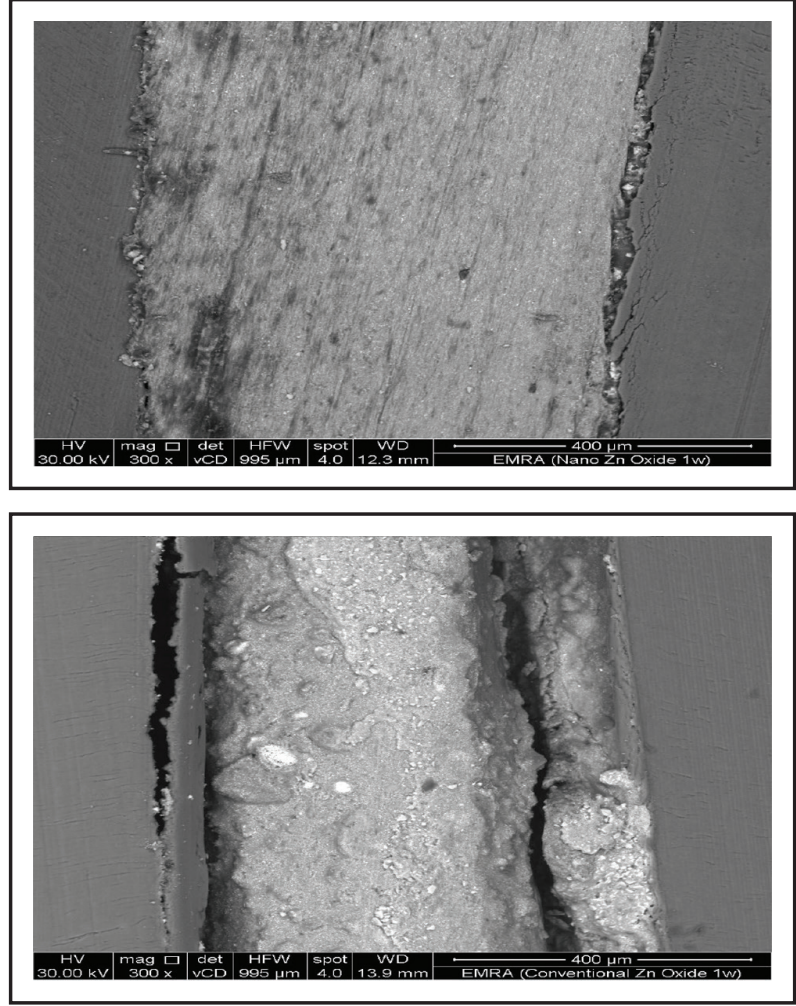

Figure (2) Zinco Oxide Nano-particles sealer Conventional Zinc Oxide sealer

\section{DISCCUSION}

The sealing of the root canal apically by the sealer is important to prevent communication of root canal contents with periapical tissue. The properties of sealers like flow, consistency, setting characteristics, solubility and adhesion to root canals are important in obtaining a hermetic seal of the root canal ${ }^{(10)}$.

$\mathrm{ZnO}$ is a safe material which has been used widely in biomedical applications Although, $\mathrm{ZnO}$ has interesting antibacterial properties which can be enhanced by doping some elements such as $\mathrm{Ni}$ and $\mathrm{Co}$ in $\mathrm{ZnO}$ matrix, $\mathrm{ZnO}$ nanostructures show better antibacterial properties due to their surface enhancement ${ }^{(11)}$.

ProTaper Universal rotary files were used for canal instrumentation, as they have a convex triangular cross section which decrease the friction between the blade of file and the canal wall and increases its cutting efficiency. Moreover, they have a continuously variable helical angle and pitch over their cutting blades which has been claimed by the manufacturer that this design optimizes it's cutting action, effectively allows it's blades to auger debris out of the canal, and importantly prevents the instruments from inadvertently screwing into the canal $^{(12)}$.

In this study the results showed that, Zinc oxide nanopowder had less leakage than the conventional zinc oxide through all periods of examination.

This results came in agreement with a study ${ }^{(13)}$, which concluded that the prepared $\mathrm{ZnO}$ and $\mathrm{ZnO}: \mathrm{Ag}$ nanopowders have a leakage less than the standard (AH26) and ZOE which are commonly used as root filling materials, It also came in haromony with another study ${ }^{(14)}$, which showed that the synthesized $\mathrm{ZnO}$ nano-powder sealers are suitable for use as a nano-sealer in root canal therapy to prevent leakage and it shows less leakage than conventional zinc oxide and AH26 sealers.

This was also confirmed by SEM observations, which revealed intimate adaptation of zinc oxide nanoparticles sealer to the dentin and gutta percha walls 
In the present study, it was very important to asses leakage not only immediately after sealing but also after some time, as the sealer in the root canal could be exposed to tissue fluid and exudates for prolonged time.Therefore, the microleakage of the two tested groups was evaluated at one week, one month and three months.

In the present study, the results showed microleakage in both Zno nano-partciles and Conventional zno sealers in one week samples which could be explained by elusion of free eugenol and unreacted zinc oxide particles from the set sealer mix ${ }^{(15)}$.

Also Polymerization shrinkage of ZOE-based sealers can lead to stress development on root canal walls, resulting in marginal gaps, microleakage, and clinical failures. Forces of polymerization shrinkage can exceed its bond strength to root dentin, permitting debonding on one side of the root canal filling to relieve stress. ${ }^{(16)}$

In the current study, the results showed that the leakage of the three tested groups decreased from one week to one month. The decrease in leakage from one week to one month may be related to volumetric expansion of the sealer in accordance with a study which reported that an initial increase in the volume of Endofill sealer started after 24 hours from mixing time, and then acquired its original volume after 21 days. ${ }^{(17)}$

In the current study, the decrease in the microleakage from one week to one month for the two tested groups was in disagreement with the results of previous study which found an increase in apical leakage of Kerr's pulp canal sealer from 24 hours to two weeks, and explained that by sealer disintegration over time. ${ }^{(18)}$

At three month observation period, the two tested groups in the current study showed significantly greater leakage in comparison to the two other testing periods. This could be attributed to sealer disintegration or sealer shrinkage as confirmed by

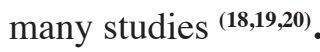

In part 2 of this study, sealing ability and adaptation of the sealers were evaluated using SEM. In the samples preparation for SEM evaluation, the steps were done following previous study ${ }^{(21)}$, The roots were sectioned longitudinally. In examination of the horizontal sections only a small number of sections can be observed and evaluated. While in the longitudinal sectioning the whole length of the root can be examined ${ }^{(\mathbf{1 8 , 2 1 )}}$.

The SEM examination of the two tested groups showed that, Zno-nano particles sealer was more penetrated to dentinal tubules than Conventional Zno sealer this may be due to its nano sized particles which allowes more penetration and sealing to dentinal tubules.

\section{CONCLUSION}

Within limits of the current study, we can conclude the following:

1- Zinc Oxide nano particles based sealer has higher sealing ability than conventional zinc oxide based sealer.

2- Nano sized particles allow more adaptation and penetration into dentinal tubules than macro sized one.

3- Sealing of the two tested sealers in the coronal and middle thirds of the root is more than the apical third when examined by SEM.

\section{REFRENCES}

1. Singh H, Markan S, Kaur M, Gupta G. Endodontic Sealers: Current concepts and comparative analysis. Dent Open J.2015; 2:32-37.

2. Wu M, van der Sluis L, Ardila C, Wesselink P. Fluid movement along the coronal two-thirds of root fillings placed by three different gutta-percha techniques. Int Endod J. 2003;36:533-40.

3. Loushine B et al. Setting properties and cytotoxicity evaluation of a premixed Bioceramic root canal sealer. J of Endod. 2011;37:673-77. 
4. Vijay R, Indira R. Effect of glass-ionomer cement as an intra-canal barrier in post space prepared teeth: An in vitro study. J Conserv Dent. 2009;12:65-68.

5. Kumar S, Shivanna V, Naian MT, Shivamurthy G. Comparative evaluation of the apical sealing ability and adaptation to dentine of three resin-based sealers: An in vitro study. J Conserv Dent. 2011; 14:16-20.

6. Huumonen S, Lenander-Lumikari M, Sigurdsson A, Ørstavik D. Healing of apical periodontitis after endodontic treatment: a comparison between a siliconebased and a zinc oxide-eugenol-based sealer. Int endod J. 2003; 36:296-301.

7. Pal S, Tak Y, Song J. Does the antibacterial activity of silver nanoparticles depend on the shape of the nanoparticle? A study of the gram-negative bacterium Escherichia coli. Appl and Environ Microbiol. 2007;73:1712-20.

8. Allaker R, Memarzadeh K. Nanoparticles and the control of oral infections. Int J of Antimicrob Agents.2014;43:95-104.

9. Kocak M, Er O, Saglam B, Yaman S. Apical leakage of epiphany root canal sealer combined with different master cones. Eur J Dent. 2008;2:91.

10. Zhou H, Shen Y, Zheng W, Li L, Zheng Y, Haapasalo M. Physical properties of five root canal sealers. J of Endod. 2013;39:1281-86.

11. Branstetter J, Von Fraunhofer J. The physical properties and sealing action of endodontic sealer cements: a review of the literature. J of Endod. 1982;8:312-16.

12. Ruddle C. The ProTaper endodontic system: geometries, features and guidelines for use. Dent Today. 2001; 20:60-67.
13. Rad M, Kompany A, Zak A, Javidi M, Mortazavi S. Microleakage and antibacterial properties of $\mathrm{ZnO}$ and ZnO: Ag nanopowders prepared via a sol-gel method for endodontic sealer application. $\mathrm{J}$ of nanoparticle res. 2013;1915:25

14. Javidi M, Zarei M, Naghavi N, Mortazavi M, Nejat A. Zinc oxide nano-particles as a sealer in endodontics and it's sealing ability. Contemp clinic dent. 2014;5:20-24.

15. Schäfer, E., and T. Zandbiglari. Solubility of root-canal sealers in water and artificial saliva, Inter Endod $\mathrm{J}$. 2003;36:660-69.

16. Kardon B, Kuttler S, Hardigan P, Dorn S. An in vitro evaluation of the sealing ability of a new root-canalobturation system. J of Endod. 2003;29:658-661.

17. Kazemi R, Safavi K, Spångberg L. Dimensional changes of endodontic sealers. Oral surgery, oral medicine, oral pathology. 1993; 76:766-71.

18. Yared G, Dagher F. Apical enlargement: influence on the sealing ability of the vertical compaction technique. $\mathrm{J}$ of Endod.1994;20:31-34.

19. Yared G, Dagher F. Sealing ability of the vertical condensation with different root canal sealers. J of Endod 1996;22:6-8

20. Bou Dagher F E, Yared G M, and Machtou P. Microleakage of new and old Kerr root canal sealers. J of Endod. 1997; 23:442-43.

21. Sevimay S, Kalayci A. Evaluation of apical sealing ability and adaptation to dentine of two resin-based sealers. J of oral rehab. 2005;32:105-10. 